\title{
Association of Serum Uric Acid with Biventricular Myocardial Dysfunction in Patients with type 2 Diabetes Mellitus: A Cross-sectional Study
}

\author{
Ju-Hua Liu \\ Devision of Cardiology, Department of Medicine, the University of Hong Kong Shenzhen Hospital \\ Mei-Zhen Wu \\ Devision of Cardiology, Department of Medicine, the University of Hongkong, Queen Mary Hospital \\ Si-Min Li \\ Devision of Cardiology, Department of MEdicine, the University of Hong Kong Shenzhen Hospital \\ Yan Chen \\ University Cardiology

\section{Qing-Wen Ren} \\ Devision of cardiology, Department of MEdicine, the University of Hong Kong Shenzhen Hospital

\section{Qing-Shan Lin} \\ Devision of Ultrasound, Department of Radiology, the University of Hong Kong Shenzhen Hospital

\section{Ming-Yen Ng} \\ Devision of Ultrasound, Department of Regiology, the University of Hong Kong Sehzhen Hospital \\ Hung-Fat Tse \\ Devision of Cardiology, Department of Medicine, the University of Hong Kong Shenshen Hospital \\ Kai-Hang Yiu ( $\nabla$ khkyiu@hku.hk) \\ University of Hong Kong
}

\section{Original investigation}

Keywords: Type 2 diabetic mellitus, Serum uric acid, Right ventricular function, Left ventricular function

Posted Date: December 23rd, 2020

DOl: https://doi.org/10.21203/rs.3.rs-130641/v1

License: (c) (i) This work is licensed under a Creative Commons Attribution 4.0 International License. Read Full License 


\section{Abstract}

Background

Increased serum uric acid (SUA) is common in patients with type 2 diabetes mellitus (T2DM) and is associated with left ventricular (LV) myocardial dysfunction. Nonetheless the association of SUA with right ventricular (RV) function in patients with T2DM has not been studied. This study aimed to investigate the association of SUA with biventricular myocardial function in patients with T2DM.

Methods

A total of 560 patients with T2DM were enrolled and divided into four groups according to quartile of SUA. Transthoracic echocardiography was performed and two-dimensional speckle tracking used to measure biventricular myocardial strain, including LV global longitudinal strain (GLS), circumferential strain (CS), radial strain (RS), and RV free wall longitudinal stra in (RV-FWLS).

Results

The absolute value of all biventricular strain parameters showed a stepwise decrease across SUA quartiles (all $P<0.01)$. In particular, LV assessment by GLS, CS and RS demonstrated that those in the $4^{\text {th }}$ quartile were impaired compared with the other quartiles (all $\mathrm{P}<0.05$ ). Similarly, RV-FWLS of the $4^{\text {th }}$ quartile was significantly impaired compared with the $1^{\text {st }}$ and $2^{\text {nd }}$ quartiles (all $\mathrm{P}<0.05$ ). The same reduction in biventricular strain across SUA quartiles was observed in patients with estimated glomerular filtration rate邓or $\geq 60 \mathrm{ml} / \mathrm{min} / 1.73 \mathrm{~m}^{2}$, and glycated hemoglobin $<$ or $\geq 7.0 \%$ (all $\left.\mathrm{P}<0.05\right)$. Multivariable linear regression analysis demonstrated that higher quartile of SUA was independently associated with impaired biventricular myocardial strain (all $\mathrm{P}<0.05$ ).

\section{Conclusions}

SUA was independently associated with biventricular myocardial dysfunction in asymptomatic T2DM patients, regardless of renal function or diabetic control.

\section{Background}

The population of patients with type 2 diabetes mellitus (T2DM) is increasing and is expected to affect an estimated 650 individuals million in 2045.[1] It is well recognized that T2DM is directly detrimental to myocardial function, independent of underlying coronary artery disease, and may lead to clinically overt heart failure.[1] Identifying factors that contribute to myocardial dysfunction is clinically crucial to better understand its pathophysiology and to help strategize treatments to prevent T2DM-related heart failure.

A high serum uric acid (SUA) level (hyperuricemia) is associated with a worsened cardiovascular outcome but can be alleviated by administration of uric acid lowering agents.[2] Numerous studies using speckle tracking-derived strain analysis have demonstrated that a high SUA is associated with subclinical left ventricular (LV) myocardial dysfunction in patients with heart failure. Right ventricular (RV) function, which is just as important as LV function in predicting clinical outcome, has been shown to also be impaired in patients with T2DM.[3-5] The role of hyperuricemia in both LV and RV (biventricular) myocardial function in patients with T2DM has nonetheless not been evaluated. The present study aimed to evaluate the relation of SUA to biventricular myocardial function, assessed by speckle tracking-derived strain analysis, in asymptomatic patients with T2DM.

\section{Methods}




\section{Study population}

A total of 1539 patients with T2DM, as defined by American Diabetes Association criteria[6], were recruited between March 2014 and March 2020 from the University of Hong Kong Shenzhen Hospital, Shenzhen, China, and Queen Mary Hospital, Hong Kong, China. Patients with severe liver or renal dysfunction $(n=86)$, a documented history of cardiovascular disease including coronary artery disease $(n=427)$, myocardial infarction $(n=210)$, hospitalization for heart failure $(n=108)$, significant valvular disease $(n=95)$ or congenital heart disease $(n=33)$ and those who refused to participate $(n=20)$ were excluded from the study. A final 560 patients were enrolled, written informed consent was obtained from all participants.

\section{Study protocol}

All patients underwent a complete physical examination and an interview to establish baseline characteristics. Blood pressure was measured after resting for at least 5 minutes. Smoking status was recorded as positive if patients had smoked (ever or current). Body weight and height were measured and body mass index (BMI) calculated in $\mathrm{kg} / \mathrm{m}^{2}$. Hypertension was defined as resting systolic or diastolic blood pressure $\geq 140$ or $90 \mathrm{mmHg}$ respectively at two clinic visits or prescription of antihypertensive medication. A fasting blood sample were obtained to measure glycated hemoglobin $(\mathrm{HbA} 1 \mathrm{c})$, fasting blood glucose (FBG), total cholesterol (TC), total triglyceride (TG), high-density lipoprotein cholesterol (HDL-c), low-density lipoprotein cholesterol (LDL-c), creatinine and SUA. Estimated glomerular filtration rate (eGFR) was obtained according to the Modification of Diet in Renal Disease (MDRD) study equations. [7] Patients were divided into four groups according to quartile of SUA.

\section{Echocardiographic measurement of biventricular structure and function}

Transthoracic echocardiographic examination was performed in all patients using a Vivid E9, General Electric Vingmed Ultrasound machine (Milwaukee, WI, USA), with the patient lying in the lateral decubitus position. A 3.5-MHz transducer was used to obtain images that were digitally stored in cine-loop format with three cardiac cycles. Off-line analysis was performed using the EchoPAC version 108.11.1(General Electric Vingmed, Horten, Norway).

The dimensions of the LV chamber were measured according to the current recommendations.[8] Inter-ventricular septal dimension at end-diastole (IVSd), LV posterior wall thickness at end-diastole (LVPWd) and LV end-diastolic dimension (LVEDd) were measured using the two-dimensional echocardiography guided M-mode approach from the LV long axis view. Relative wall thickness (RWT) was defined as the ratio of ( $2 \times$ LVPWDd)/LVEDd. LV mass (LVM) was calculated as follows: LVM $(\mathrm{g})=0.8 \times\left[1.04 \times(\text { LVEDd + LVPWDd + IVSDd })^{3}-(\text { LVEDd })^{3}\right]+0.6$. LV mass index $($ LVMI) was then calculated based on LVM/body surface area $\left(\mathrm{g} / \mathrm{m}^{2}\right)$. LV volume at the end of diastole (LVEDV), LV volume at the end of systole (LVESV) and left ventricular ejection fraction (LVEF) were determined from apical four and two-chamber views using a modified Simpson's biplane method. Peak velocity in early (E-wave) and late (A-wave) diastole of mitral valve inflow was measured by pulsed-wave Doppler of the mitral valve inflow in apical four-chamber view and E/A ratio calculated. Pulsed wave tissue doppler imaging was used to measure early diastolic velocity of the mitral valve annulus with the sample volume placed at the septum (E'-sep) and lateral (E'-lat) annulus of the mitral valve, and average E/E' calculated. [9]

Conventional echocardiographic parameters of RV function were measured according to current recommendations. [8] The RV end-diastolic area (RVEDA) and RV end-systolic area (RVESA) were determined in the RV-focused apical four chamber view, and percentage of RV fractional area change calculated (RV-FAC) $=($ RVEDA-RVESA)/ RVEDA $\times 100$. Tricuspid annular plane systolic excursion (TAPSE) was calculated as an index of RV longitudinal systolic function by placing an M-mode cursor through the tricuspid annulus in the apical four-chamber view and measuring the difference between end-diastolic and end-systolic longitudinal motion of the annulus. RV systolic pressure (RVSP) was calculated as the sum of the estimated right atrial pressure and the peak pressure gradient between the peak right ventricle and right atrium, as 
estimated using the simplified Bernoulli equation for peak velocity represented by the tricuspid regurgitation Doppler signal.[10]

\section{D speckle tracking strain analyses of biventricular function}

Two-dimensional speckle tracking echocardiography was used to measure biventricular myocardial strain. LV global longitudinal strain (GLS) was measured from the three apical views: two chamber view, four chamber view and long-axis view. Each wall was subsequently divided into three levels (basal, middle and apical) and a total of 18 segmental strain curves obtained. GLS was calculated as the mean peak systolic strain value of the 18 segments. Circumferential strain (CS) and radial strain (RS) were measured from the LV short-axis view at the papillary muscle level, and were derived from the average peak systolic strain value of six segments. [11] Two-dimensional speckle tracking-derived RV free wall longitudinal strain (RV-FWLS) was measured from an RV-focused apical four chamber view. RV-FWL was calculated manually by taking the mean of the three segments forming the RV free wall (basal, middle and apical). [8, 12] BlandAltman and intraclass correlation coefficient revealed satisfactory correlations for inter- and intra-observer variability for biventricular myocardial strain, including GLS, CS, RS and RV-FWLS, in 20 randomly selected patients (Supplementary Table 1).

\section{Statistical analysis}

Data are expressed as mean \pm standard deviation for continuous variables with normal distribution, median (interquartile range) for those with skewed distribution, and frequencies (proportions) for categorical variables. Continuous variables were compared using one-way analysis of variance with post hoc analysis by Bonferroni for normally distributed data, and Mann-Whitney $U$ test for parameters with skewed distribution. Multivariate linear regression analyses were performed to investigate the association between quartile of SUA and biventricular myocardial strain by adjusted variables that were statistically significant in univariate analyses. Statistical analyses were performed using SPSS (version 22.0) for windows, and $\mathrm{P}<0.05$ considered to indicate statistical significance.

\section{Results}

\section{Clinical characteristics of study patients}

The main demographic characteristics of the study population are shown in Table 1. The mean age of patients was $60 \pm$ 11 years and $53.2 \%$ were male. Mean duration of diabetes was 7 years and mean $\mathrm{HbA} 1 \mathrm{c}$ was $7.5 \pm 1.6 \%$. Prevalence of hypertension and dyslipidemia was $82.5 \%$ and $50.9 \%$, respectively. The median circulating SUA level was 347.6 (285.0429.5) $\mathrm{umol} / \mathrm{L}$ and patients were divided subsequently into four groups according to quartile of SUA: 1 st quartile SUA< 285.0umol/L, 2nd quartile $285.0 \leq \mathrm{SUA} \varangle 347.6 \mathrm{umol} / \mathrm{L}$, 3rd quartile $347.6 \leq \mathrm{SUA} \Downarrow 429.5 \mathrm{umol} / \mathrm{L}$, and 4th quartile SUA $\geq$ 429.5umol/L. Additionally, those with higher quartile of SUA were more likely to be male and a smoker with a higher BMI, diastolic blood pressure, serum TG, and hypertension and a lower eGFR (all P<0.05). Age, systolic blood pressure, prevalence of hyperlipidemia, duration of diabetes, fasting glucose level and lipid profile except TG nevertheless showed no significant difference across SUA quartiles (all P >0.05). 
Table 1

Clinical characteristics of patients according to quartile of serum uric acid

\begin{tabular}{|c|c|c|c|c|c|c|}
\hline $\begin{array}{l}\text { Serum uric acid } \\
\text { (umol/L) }\end{array}$ & $\begin{array}{l}\text { Total }(n=560) \\
347.6(285.0-429.5)\end{array}$ & $\begin{array}{l}\text { 1st Quartile }(\mathrm{n}= \\
\text { 138) }<285.0\end{array}$ & $\begin{array}{l}\text { 2nd Quartile } \\
(n=142) \\
285.0-347.6\end{array}$ & $\begin{array}{l}\text { 3rd } \\
\text { Quartile(n } \\
=140) \\
\\
347.6- \\
429.5\end{array}$ & $\begin{array}{l}\text { 4th Quartile }(n= \\
140) \geq 429.5\end{array}$ & $\mathbf{P}$ \\
\hline \multicolumn{7}{|c|}{ Baseline clinical database } \\
\hline Age (years) & $60 \pm 11$ & $60 \pm 10$ & $59 \pm 11$ & $59 \pm 11$ & $61 \pm 13$ & 0.38 \\
\hline Male, n (\%) & $298(53.2)$ & $60(43.5)$ & $67(47.2)$ & $88(62.9)^{\S^{*}}$ & $83(53.9)^{\dagger \# \&}$ & $<01$ \\
\hline $\mathrm{BMI}\left(\mathrm{kg} / \mathrm{m}^{2}\right)$ & $25.8 \pm 4.3$ & $24.8 \pm 4.3$ & $24.9 \pm 3.3$ & $26.1 \pm 3.3$ & $27.4 \pm 5.4^{\dagger \#}$ & $\begin{array}{l}< \\
0.01\end{array}$ \\
\hline $\mathrm{SBP}(\mathrm{mmHg})$ & $144 \pm 25$ & $141 \pm 25$ & $142 \pm 24$ & $145 \pm 24$ & $149 \pm 27$ & 0.05 \\
\hline $\mathrm{DBP}(\mathrm{mmHg})$ & $84 \pm 13$ & $80 \pm 12$ & $84 \pm 13$ & $85 \pm 14 \S$ & $86 \pm 13^{\S}$ & ¿́.01 \\
\hline \multicolumn{7}{|l|}{ Medical history } \\
\hline $\begin{array}{l}\text { Hypertension, } n \\
(\%)\end{array}$ & $462(82.5)$ & $101(73.2)$ & 115(81.0) & $124(88.6) *$ & $122(87.1) \#$ & <. 01 \\
\hline $\begin{array}{l}\text { Hyperlipidemia, n } \\
(\%)\end{array}$ & $285(50.9))$ & $67(48.6)$ & $66(46.5)$ & $82(58.6)$ & $70(50.0)$ & 0.20 \\
\hline Smoker, n (\%) & $120(21.4)$ & $22(15.9)$ & $23(16.2)$ & $39(27.9)$ & $36(25.7)$ & 0.02 \\
\hline $\begin{array}{l}\text { Diabetes duration } \\
\text { (years) }\end{array}$ & $7.0(2.0-12.0)$ & $8.0(3.0-15.0)$ & $\begin{array}{l}8.5(2.0- \\
14.3)\end{array}$ & $\begin{array}{l}6.0(3.0- \\
10.0)\end{array}$ & $6.5(2.0-10.0)$ & 0.10 \\
\hline \multicolumn{7}{|c|}{ Serum glucose level, lipid profile, and renal function } \\
\hline FBG (mmol/L) & $8.09 \pm 2.98$ & $8.47 \pm 3.82$ & $8.35 \pm 2.95$ & $7.69 \pm 2.18$ & $7.83 \pm 2.68$ & 0.12 \\
\hline HbA1C (\%) & $7.53 \pm 1.56$ & $7.79 \pm 1.80$ & $7.60 \pm 1.46$ & $7.30 \pm 1.34$ & $7.43 \pm 1.59$ & 0.07 \\
\hline TG (mmol/L) & $1.55(1.08-2.29)$ & $\begin{array}{l}1.40(0.98- \\
2.31)\end{array}$ & $\begin{array}{l}1.39(1.00- \\
2.11)\end{array}$ & $\begin{array}{l}1.60(1.17- \\
2.18)^{\star}\end{array}$ & $\begin{array}{l}1.69(1.29- \\
2.59)^{\dagger \#}\end{array}$ & $\begin{array}{l}<.01 \\
0.01\end{array}$ \\
\hline $\mathrm{TC}(\mathrm{mmol} / \mathrm{L})$ & $4.41 \pm 1.20$ & $4.29 \pm 1.24$ & $4.52 \pm 1.16$ & $4.24 \pm 1.14$ & $4.60 \pm 1.24$ & 0.05 \\
\hline HDL-c (mmol/L) & $1.21 \pm 0.38$ & $1.24 \pm 0.37$ & $1.26 \pm 0.39$ & $1.15 \pm 0.27$ & $1.20 \pm 0.47$ & 0.09 \\
\hline
\end{tabular}

Abbreviations: BMI, body mass index; DBP, diastolic blood pressure; eGFR, estimated glomerular filtration rate; FBG, fasting blood glucose; HbA1c, glycated hemoglobin; HDL-c, high-density lipoprotein cholesterol; LDL-c, low-density lipoprotein cholesterol; SBP, systolic blood pressure; TC, total cholesterol; TG, total triglyceride.

9 $\mathrm{P}<0.05$ between 1 st quartile and 2 nd quartile

$\S \mathrm{P}<0.05$ between 1 st quartile and 3rd quartile

† $\mathrm{P}<0.05$ between 1 st quartile and 4 th quartile

* $P<0.05$ between 2 nd quartile and 3rd quartile

\# $\mathrm{P}<0.05$ between 2 nd quartile and 4 th quartile

$\& P<0.05$ between 3rd quartile and 4 th quartile 


\begin{tabular}{|c|c|c|c|c|c|c|}
\hline $\begin{array}{l}\text { Serum uric acid } \\
\text { (umol/L) }\end{array}$ & $\begin{array}{l}\text { Total }(n=560) \\
347.6(285.0-429.5)\end{array}$ & $\begin{array}{l}\text { 1st Quartile(n= } \\
\text { 138)<285.0 }\end{array}$ & $\begin{array}{l}\text { 2nd Quartile } \\
(n=142) \\
285.0-347.6\end{array}$ & $\begin{array}{l}\text { 3rd } \\
\text { Quartile(n } \\
=140) \\
347.6- \\
429.5\end{array}$ & $\begin{array}{l}\text { 4th Quartile }(n= \\
140) \geq 429.5\end{array}$ & $\mathbf{P}$ \\
\hline LDL-c (mmol/L) & $2.74 \pm 1.04$ & $2.70 \pm 0.99$ & $2.82 \pm 1.08$ & $2.62 \pm 0.96$ & $2.82 \pm 1.14$ & 0.33 \\
\hline $\begin{array}{l}\text { eGFR } \\
\left(\mathrm{ml} / \mathrm{min} / 1.73 \mathrm{~m}^{2}\right)\end{array}$ & $82.3 \pm 25.6$ & $90.1 \pm 27.2$ & $87.7 \pm 21.3$ & $82.4 \pm 22.1$ & $68.8 \pm 26.4^{\dagger \# \&}$ & $\hat{0.01}$ \\
\hline \multicolumn{7}{|c|}{$\begin{array}{l}\text { Abbreviations: BMI, body mass index; DBP, diastolic blood pressure; eGFR, estimated glomerular filtration rate; FBG, } \\
\text { fasting blood glucose; HbA1c, glycated hemoglobin; HDL-c, high-density lipoprotein cholesterol; LDL-c, low-density } \\
\text { lipoprotein cholesterol; SBP, systolic blood pressure; TC, total cholesterol; TG, total triglyceride. }\end{array}$} \\
\hline \multicolumn{7}{|c|}{ 9 $\mathrm{P}<0.05$ between 1 st quartile and 2 nd quartile } \\
\hline \multicolumn{7}{|c|}{$\S \mathrm{P}<0.05$ between 1 st quartile and 3rd quartile } \\
\hline \multicolumn{7}{|c|}{$+P<0.05$ between 1 st quartile and 4 th quartile } \\
\hline \multicolumn{7}{|c|}{ * $P<0.05$ between 2 nd quartile and 3rd quartile } \\
\hline \multicolumn{7}{|c|}{$\# P<0.05$ between 2 nd quartile and 4 th quartile } \\
\hline$\& P<0.05$ between & Brd quartile and 4 th qu & artile & & & & \\
\hline
\end{tabular}

\section{Comparison of biventricular conventional echocardiography parameters according to quartile of SUA}

In the present study, biventricular structure and function showed a significant difference according to quartile of SUA (Table 2). Patients with a higher quartile SUA had greater LV wall thickness and LV volume, decreased LVEF, and impaired LV diastolic function compared with those with lower quartile SUA (all P<0.05). When these parameters were compared between different quartiles, patients with the highest quartile SUA (4th quartile) had a higher IVSd, LVPWd, RWT, average $E / E^{\prime}$, and lower LVEF, E'-sep and E'-lat (4th quartile vs 1st quartile, or 4th quartile vs 2 nd quartile, all $P<0.05$ ). All aforementioned parameters of LV structure and function nonetheless showed no difference between the $1 \mathrm{st}$ and $2 \mathrm{nd}$ quartile, or the 3rd and 4th quartile (all $\mathrm{P}>0.05$ ). 
Table 2

Comparison of biventricular conventional echocardiography parameters according to quartile of serum uric acid

\begin{tabular}{|c|c|c|c|c|c|}
\hline Variable & $\begin{array}{l}\text { 1st Quartile }(n= \\
138)\end{array}$ & $\begin{array}{l}\text { 2nd Quartile ( } n= \\
\text { 142) }\end{array}$ & $\begin{array}{l}\text { 3rd Quartile }(n= \\
140)\end{array}$ & $\begin{array}{l}\text { 4th Quartile }(n= \\
140)\end{array}$ & $\mathbf{P}$ \\
\hline \multicolumn{6}{|l|}{ LV function } \\
\hline IVSd (mm) & $9.91 \pm 1.65$ & $10.01 \pm 1.88$ & $10.84 \pm 2.08 \S^{*}$ & $10.73 \pm 2.02^{\dagger \#}$ & $\dot{0} 01$ \\
\hline LVPWd (mm) & $9.89 \pm 1.43$ & $9.94 \pm 1.52$ & $10.61 \pm 1.58 \S^{\star}$ & $10.68 \pm 1.78^{\dagger \#}$ & ¿́.01 \\
\hline RWT & $0.44 \pm 0.07$ & $0.44 \pm 0.07$ & $0.46 \pm 0.08$ & $0.47 \pm 0.09^{\dagger}$ & $\hat{0.01}$ \\
\hline LVMI $\left(\mathrm{g} / \mathrm{m}^{2}\right)$ & $87.7 \pm 21.54$ & $87.7 \pm 23.7$ & $95.4 \pm 25.1$ & $96.6 \pm 32.3$ & 0.01 \\
\hline LVEDV (ml) & $95.4 \pm 20.1$ & $95.6 \pm 22.5$ & $100.6 \pm 23.8$ & $101.5 \pm 24.3$ & 0.04 \\
\hline LVESV (ml) & $33.5 \pm 10.6$ & $33.3 \pm 10.1$ & $37.3 \pm 12.9 \S^{*}$ & $37.0 \pm 12.9$ & $\dot{0} 01$ \\
\hline LVEF (\%) & $66.0 \pm 6.1$ & $65.7 \pm 5.4$ & $63.9 \pm 5.5^{\S}$ & $63.7 \pm 6.8^{\dagger \#}$ & $\dot{0} 01$ \\
\hline $\mathrm{E} / \mathrm{A}$ & $0.92 \pm 0.28$ & $0.88 \pm 0.26$ & $0.86 \pm 0.27$ & $0.89 \pm 0.46$ & 0.57 \\
\hline$E^{\prime}-\operatorname{sep}(\mathrm{mm} / \mathrm{s})$ & $7.55 \pm 2.46$ & $7.36 \pm 2.54$ & $6.98 \pm 2.30$ & $6.67 \pm 2.29^{\dagger}$ & 0.01 \\
\hline$E^{\prime}-$ lat $(\mathrm{mm} / \mathrm{s})$ & $9.65 \pm 2.68$ & $10.06 \pm 2.73$ & $9.08 \pm 2.62^{\star}$ & $9.04 \pm 2.92^{\#}$ & 0.01 \\
\hline Average $E / E^{\prime}$ & $9.5 \pm 3.0$ & $9.3 \pm 3.3$ & $9.7 \pm 4.1$ & $10.6 \pm 5.0^{\#}$ & 0.03 \\
\hline \multicolumn{6}{|l|}{ RV function } \\
\hline RVEDA $\left(\mathrm{cm}^{2}\right)$ & $12.5 \pm 2.1$ & $12.5 \pm 2.4$ & $12.5 \pm 2.7$ & $12.1 \pm 2.5$ & 0.43 \\
\hline RVESA $\left(\mathrm{cm}^{2}\right)$ & $6.42 \pm 1.18$ & $6.47 \pm 1.27$ & $6.76 \pm 1.58$ & $6.88 \pm 1.92$ & 0.04 \\
\hline RV-FAC (\%) & $48.6 \pm 6.7$ & $47.8 \pm 7.4$ & $45.7 \pm 8.1 \S$ & $43.2 \pm 8.0^{\dagger \# \&}$ & $\hat{0} .01$ \\
\hline TAPSE (mm) & $21.1 \pm 3.3$ & $20.8 \pm 2.9$ & $20.0 \pm 3.51^{\S}$ & $18.8 \pm 3.1^{\dagger \# \&}$ & $\hat{0.01}$ \\
\hline $\begin{array}{l}\text { RVSP } \\
(\mathrm{mmHg})\end{array}$ & $27.9 \pm 8.1$ & $26.9 \pm 7.1$ & $26.6 \pm 6.5$ & $28.9 \pm 9.5$ & 0.30 \\
\hline \multicolumn{6}{|c|}{$\begin{array}{l}\text { Abbreviations: A, peak velocity in late diastole of mitral valve inflow; E, peak velocity in early diastole of mitral valve } \\
\text { inflow; E'-sep, early diastolic velocity of mitral valve annulus at septum; E'-lat, early diastolic velocity of mitral valve } \\
\text { annulus at lateral; IVSd, inter-ventricular septal dimension at end-diastole; LV, Left ventricular; LVEDV, LV volume at the } \\
\text { end of diastolic; LVEF, LV ejection fraction; LVESV, LV volume at the end of systolic; LVMI, LV mass index; LVPWd, LV } \\
\text { posterior wall thickness at end-diastole; RV, right ventricular; RVEDA, RV end-diastolic area; RVESA, RV end-systolic } \\
\text { area; RV-FAC, RV fractional area change; RVSP, RV systolic pressure; RWT, relative wall thickness; TAPSE, tricuspid } \\
\text { annular plane systolic excursion. }\end{array}$} \\
\hline \multicolumn{6}{|c|}{ 9 $\mathrm{P}<0.05$ between 1 st quartile and 2 nd quartile } \\
\hline \multicolumn{6}{|c|}{$\S \mathrm{P}<0.05$ between 1 st quartile and 3 rd quartile } \\
\hline \multicolumn{6}{|c|}{$+\mathrm{P}<0.05$ between 1 st quartile and 4 th quartile } \\
\hline * $P<0.05$ betw & n 2nd quartile an & dartile & & & \\
\hline
\end{tabular}




\begin{tabular}{|c|c|c|c|c|c|}
\hline Variable & $\begin{array}{l}\text { 1st Quartile }(n= \\
138)\end{array}$ & $\begin{array}{l}\text { 2nd Quartile ( } n= \\
\text { 142) }\end{array}$ & $\begin{array}{l}\text { 3rd Quartile ( } n= \\
140)\end{array}$ & $\begin{array}{l}\text { 4th Quartile }(n= \\
140)\end{array}$ & $\mathbf{P}$ \\
\hline \multicolumn{6}{|c|}{$\# \mathrm{P}<0.05$ between 2 nd quartile and 4 th quartile } \\
\hline
\end{tabular}

Similarly, RV systolic function, measured by RV-FAC and TAPSE, was impaired in patients with higher quartiles of SUA compared with those with lower quartiles (all P<0.05). RV-FAC and TAPSE decreased in a progressive manner across SUA quartile with the lowest values in the 4th quartile (all $P<0.05$ ). Nevertheless, both RV-FAC and TAPSE showed no significant difference between the 1 st and 2 nd quartile (all $P>0.05$ ).

\section{Association of quartile of SUA with biventricular myocardial strain}

Comparison of biventricular strain in T2DM patients according to quartile of SUA is shown in Table 3. Consistent with the results of conventional parameters of biventricular function, the absolute value of all biventricular strain parameters showed a stepwise decrease across SUA quartiles (all P<0.01) (Table 3), showing significantly worse values for GLS, CS and RS in the 4th quartile compared with the other three (all $\mathrm{P}<0.05$ ). The same trend was observed for RV-FWLS that was significantly impaired in the 4th quartile compared with the first two quartiles (all $\mathrm{P}<0.05$ ). None of the biventricular strain parameters showed any difference between the 1st and 2nd quartile (all $P>0.05$ ). Univariate linear regression further demonstrated that SUA of the 3rd quartile and 4th quartile was associated with impaired biventricular myocardial strain (all $P<0.05)$ (Table 4). Multivariate linear regression, adjusted for factors that were statistically significant in univariate analyses, demonstrated that higher quartile of SUA remained an independent factor associated with impaired biventricular myocardial strain (all $\mathrm{P}<0.05)($ Table 5).

Table 3

Comparison of biventricular strain according to quartile of serum uric acid

\begin{tabular}{|c|c|c|c|c|c|}
\hline Variable & $\begin{array}{l}\text { 1st Quartile ( } n= \\
138)\end{array}$ & $\begin{array}{l}\text { 2nd Quartile ( } n= \\
\text { 142) }\end{array}$ & $\begin{array}{l}\text { 3rd Quartile }(n= \\
140)\end{array}$ & $\begin{array}{l}\text { 4th Quartile ( } n= \\
\text { 140) }\end{array}$ & $\mathbf{P}$ \\
\hline GLS (\%) & $-21.1 \pm 3.6$ & $-20.6 \pm 3.0$ & $-19.3 \pm 3.3^{\S^{\star}}$ & $-17.7 \pm 3.7^{\dagger \# \&}$ & $<0.01$ \\
\hline CS (\%) & $-19.3 \pm 3.6$ & $-19.5 \pm 3.7$ & $-17.3 \pm 3.7 \S^{\star}$ & $-16.0 \pm 3.6^{\dagger \# \&}$ & $<0.01$ \\
\hline RS (\%) & $35.9 \pm 11.5$ & $34.7 \pm 10.1$ & $31.7 \pm 12.8 \S$ & $27.5 \pm 9.8^{\dagger \# \&}$ & $<0.01$ \\
\hline $\begin{array}{l}\text { RV-FWLS } \\
(\%)\end{array}$ & $-22.3 \pm 4.9$ & $-21.2 \pm 5.1$ & $-20.0 \pm 5.0 \S$ & $-18.3 \pm 5.4^{\dagger \#}$ & $<0.01$ \\
\hline \multicolumn{6}{|c|}{$\begin{array}{l}\text { Abbreviations: CS, circumferential strain; GLS, global longitudinal strain; RS, radial strain; RV-FWLS, right ventricular } \\
\text { free wall longitudinal strain. }\end{array}$} \\
\hline \multicolumn{6}{|c|}{ १ P $<0.05$ between 1 st quartile and 2 nd quartile } \\
\hline \multicolumn{6}{|c|}{$\S \mathrm{P}<0.05$ between 1 st quartile and 3 rd quartile } \\
\hline \multicolumn{6}{|c|}{$+P<0.05$ between 1 st quartile and 4 th quartile } \\
\hline \multicolumn{6}{|c|}{ * $P<0.05$ between 2 nd quartile and 3rd quartile } \\
\hline \multicolumn{6}{|c|}{$\# \mathrm{P}<0.05$ between 2 nd quartile and 4 th quartile } \\
\hline \multicolumn{6}{|c|}{$\& \mathrm{P}<0.05$ between 3 rd quartile and 4 th quartile } \\
\hline
\end{tabular}


Table 4

Univariate linear regression showing variables associated with biventricular strain

\begin{tabular}{|c|c|c|c|c|c|c|c|c|}
\hline \multirow[t]{2}{*}{ Variable } & \multicolumn{2}{|l|}{ GLS } & \multicolumn{2}{|l|}{ CS } & \multicolumn{2}{|l|}{ RS } & \multicolumn{2}{|c|}{ RV-FWLS } \\
\hline & $\beta$ & $\mathbf{P}$ & $\beta$ & $\mathbf{P}$ & $\beta$ & $\mathbf{P}$ & $\beta$ & $P$ \\
\hline \multicolumn{9}{|l|}{ Serum uric acid quartiles } \\
\hline 1st Quartile & \multicolumn{2}{|c|}{ Reference } & \multicolumn{2}{|c|}{ Reference } & \multicolumn{2}{|c|}{ Reference } & \multicolumn{2}{|c|}{ Reference } \\
\hline 2nd Quartile & 0.51 & 0.21 & -0.13 & 0.78 & -1.18 & 0.40 & 1.05 & 0.02 \\
\hline 3rd Quartile & 1.72 & $<0.01$ & 2.02 & $<0.01$ & -4.21 & $<0.01$ & 2.24 & $<0.01$ \\
\hline 4th Quartile & 3.42 & $<0.01$ & 3.35 & $<0.01$ & -8.35 & $<0.01$ & 3.94 & $<0.01$ \\
\hline Age (years) & 0.86 & $<0.01$ & 0.98 & 0.02 & -0.83 & 0.01 & 0.03 & 0.12 \\
\hline Gender & 0.55 & 0.08 & 0.67 & 0.05 & -0.69 & 0.50 & 0.36 & 0.46 \\
\hline BMI $\left(\mathrm{kg} / \mathrm{m}^{2}\right)$ & 0.08 & 0.04 & 0.11 & 0.02 & -0.16 & 0.25 & 0.28 & $<0.01$ \\
\hline Smoker & 0.91 & 0.02 & 0.84 & 0.04 & -1.43 & 0.25 & -0.34 & 0.55 \\
\hline Hypertension & 0.83 & 0.04 & 1.06 & 0.02 & 0.31 & 0.82 & 1.83 & $<0.01$ \\
\hline Hyperlipidemia & -0.23 & 0.46 & -0.31 & 0.37 & -2.25 & 0.03 & -0.54 & 0.26 \\
\hline Diabetes duration (years) & -0.04 & 0.07 & -0.06 & 0.02 & -0.04 & 0.53 & -0.02 & 0.52 \\
\hline HbA1c (\%) & 0.24 & 0.02 & 0.93 & 0.01 & -0.09 & 0.79 & 0.23 & 0.13 \\
\hline $\mathrm{TC}(\mathrm{mmol} / \mathrm{L})$ & 0.11 & 0.43 & 0.11 & 0.45 & 0.10 & 0.81 & 0.02 & 0.94 \\
\hline $\mathrm{TG}(\mathrm{mmol} / \mathrm{L})$ & 0.07 & 0.38 & 0.10 & 0.28 & -0.50 & 0.06 & -0.01 & 0.98 \\
\hline HDL-c (mmol/L) & -0.70 & 0.09 & -1.47 & $<0.01$ & 0.73 & 0.59 & -0.75 & 0.24 \\
\hline LDL-c (mmol/L) & 0.11 & 0.48 & 0.15 & 0.39 & 0.58 & 0.26 & 0.22 & 0.36 \\
\hline eGFR $\left(\mathrm{ml} / \mathrm{min} / 1.73 \mathrm{~m}^{2}\right)$ & -0.03 & $<0.01$ & -0.02 & $<0.01$ & 0.04 & 0.08 & -0.02 & 0.10 \\
\hline
\end{tabular}


Table 5

Multiple linear regression showing association of quartile of serum uric acid with biventricular strain

\begin{tabular}{|c|c|c|c|c|c|c|c|c|}
\hline \multirow[t]{2}{*}{ Variable } & \multicolumn{2}{|l|}{ GLS } & \multicolumn{2}{|l|}{ CS } & \multicolumn{2}{|l|}{ RS } & \multicolumn{2}{|l|}{ RV-FWLS } \\
\hline & Standardized $\beta$ & $\mathbf{P}$ & Standardized $\beta$ & $\mathbf{P}$ & $\begin{array}{l}\text { Standardized } \\
\beta\end{array}$ & $\mathbf{P}$ & $\begin{array}{l}\text { Standardized } \\
\boldsymbol{\beta}\end{array}$ & $\mathbf{P}$ \\
\hline \multicolumn{9}{|c|}{ Serum uric acid quartiles } \\
\hline 1st Quartile & Reference & & Reference & & Reference & & Reference & \\
\hline 2nd Quartile & 0.10 & 0.46 & -0.15 & 0.28 & -0.13 & 0.35 & 0.10 & 0.53 \\
\hline 3rd Quartile & 0.33 & 0.02 & 0.46 & $\begin{array}{l}<.01 \\
0.01\end{array}$ & -0.42 & $\leq .01$ & 0.26 & 0.10 \\
\hline 4th Quartile & 0.72 & $\begin{array}{l}<.01 \\
0.01\end{array}$ & 0.77 & $\begin{array}{l}<.01 \\
0.01\end{array}$ & -0.84 & $\begin{array}{l}< \\
0.01\end{array}$ & 0.47 & 0.02 \\
\hline Age (years) & 0.02 & 0.64 & 0.04 & 0.41 & -0.06 & 0.23 & 0.11 & 0.10 \\
\hline BMI $\left(\mathrm{kg} / \mathrm{m}^{2}\right)$ & -0.01 & 0.92 & 0.01 & 0.81 & 0.01 & 0.79 & 0.13 & 0.03 \\
\hline Smoker & 0.09 & 0.42 & 0.15 & 0.21 & -0.02 & 0.85 & -0.12 & 0.37 \\
\hline Hypertension & 0.06 & 0.63 & 0.09 & 0.53 & 0.11 & 0.42 & 0.10 & 0.52 \\
\hline Hyperlipidemia & -0.02 & 0.87 & -0.03 & 0.77 & -0.25 & 0.01 & -0.14 & 0.24 \\
\hline $\begin{array}{l}\text { Diabetes duration } \\
\text { (years) }\end{array}$ & -0.15 & $\dot{0} 01$ & -0.15 & $\begin{array}{l}<.01 \\
0.01\end{array}$ & -0.03 & 0.50 & -0.07 & 0.25 \\
\hline HbA1c (\%) & 0.12 & 0.02 & 0.02 & 0.74 & 0.01 & 0.86 & 0.07 & 0.24 \\
\hline HDL-c (mmol/L) & -0.03 & 0.52 & -0.09 & 0.07 & -0.02 & 0.72 & -0.04 & 0.43 \\
\hline $\begin{array}{l}\text { eGFR } \\
\left(\mathrm{ml} / \mathrm{min} / 1.73 \mathrm{~m}^{2}\right)\end{array}$ & -0.12 & 0.02 & -0.05 & 0.37 & -0.05 & 0.39 & -0.03 & 0.68 \\
\hline
\end{tabular}

Patients were further stratified into those with and without renal impairment, defined as eGFR $<$ or $\geq 60 \mathrm{ml} / \mathrm{min} / 1.73 \mathrm{~m}{ }^{2}$ respectively, and diabetic control according to $\mathrm{HbA} 1 \mathrm{c}<$ or $\geq 7 \%$. A reduced trend of biventricular strain across SUA quartiles was observed regardless of renal function or diabetic control (all $P<0.05$ ) and the absolute values of GLS, CS, RS and RV-FWLS in the 4th quartile were lower compared with the other quartiles (Supplementary Table 2 and Table 3).

\section{Discussion}

The present study demonstrates that SUA level correlates with both LV and RV dysfunction, measured by speckle tracking derived strain, in asymptomatic patients with T2DM. The association persisted when patients were stratified into those with eGFR $<$ or $\geq 60 \mathrm{ml} / \mathrm{min} / 1.73 \mathrm{~m}^{2}$, and $\mathrm{HbA} 1 \mathrm{c}<$ or $\geq 7 \%$, indicating that an effect of SUA on myocardial function is independent of renal function and diabetic control. 
Amongst patients with T2DM, the prevalence of heart failure is $22 \%$. The two conditions often coexist with bidirectional effects in terms of causation and outcome.[13] It is recognized that diabetes causes myocardial dysfunction in the absence of major epicardial coronary artery disease, termed diabetic cardiomyopathy. Imaging studies demonstrate that typical abnormalities include the presence of systolic or diastolic dysfunction with left ventricular hypertrophy. The underlying mechanism of adverse LV remodeling and myocardial dysfunction in patients with T2DM is complex and likely to be multifactorial but has nonetheless not been elucidated. Echocardiography is a readily available imaging modality that enables the detection of adverse LV remodeling and diastolic dysfunction in patients with T2DM.[14] Using echocardiography, cross-sectional studies have illustrated that oxidative stress[15], autonomic dysfunction[16], microvascular disease[17], obesity[18] and poor glycemic control[19] are possible factors associated with LV hypertrophy and diastolic dysfunction in patients with T2DM. In a recent study that involved a general population with normal LV ejection fraction, an elevated SUA was independently correlated with LV GLS, independent of cardiovascular risk factors. [20] A similar association was noted in both hypertensive and non-hypertensive patients where myocardial function, assessed by 3-dimensional speckle tracking derived strain, was correlated with SUA level.[21] Possible explanations of the association between hyperuricemia and myocardial function include the potential for elevated SUA to cause myocardial fibrosis.[22] A high SUA can also induce a chronic inflammatory state[23] and impede microcirculation[24] that further impairs myocardial contractility. Our result extends these observations and demonstrates that a high SUA contributes to LV myocardial dysfunction in patients with T2DM, a disease entity that significantly contributes to myocardial dysfunction. The validity of our observation is further established by the adjustment for renal function, $\mathrm{HbA} 1 \mathrm{c}$ level and use of medication, such as angiotensin converting enzyme inhibitors, all confounding factors that affect myocardial dysfunction in patients with T2DM. Consequently, the result supports a close association of hyperuricemia with LV myocardial dysfunction in patients with T2DM and no history of cardiovascular disease.

The importance of RV function assessment has gained considerable attention recently. As well as its correlation with LV dysfunction, it is a powerful predictor of adverse outcome.[3] Although studies have mostly focused on the assessment of LV dysfunction, there is a paucity of information regarding the role of SUA in RV function. The advent of speckle tracking derived strain analysis, with less angle dependency and superior reproducibility than conventional assessments such as TAPSE or RV-FAC, has now been validated as a promising means to evaluate RV systolic function.[25] In patients with T2DM, RV strain was significantly more impaired compared with controls and correlated with age and BMI. The same associations were noted in our result.[26] The present study further extends these correlations and is the first to demonstrate that SUA independently affects both LV and RV myocardial dysfunction in a group of otherwise asymptomatic T2DM patients. This finding not only highlights a potential mechanistic role of SUA in biventricular dysfunction, but also advocates that concomitant hyperuricemia in patients with T2DM may suggest the presence of subclinical myocardial dysfunction.

Impaired renal function, a common phenomenon in both T2DM and hyperuricemia, is known to affect myocardial function. It is thus relevant to consider the impact of renal function when evaluating the association between T2DM and hyperuricemia. Our finding confirms that a high SUA correlates closely with biventricular dysfunction, even when patients were stratified into those with and without renal dysfunction. Although it has been shown that renal dysfunction impairs myocardial function in patients with T2DM, the assessment of serum SUA may further identify those with worse cardiac function. In the same context, our result confirms that a higher SUA correlates with biventricular dysfunction, in patients with $\mathrm{HbA} 1 \mathrm{C}<$ and $\geq 7 \%$. Indeed, diabetic control, measured by $\mathrm{HbA} 1 \mathrm{c} \%$ is closely related to heart failure risk in T2DM[27] and a $\mathrm{HbA} 1 \mathrm{c} \geq 7 \%$ appears to be associated with an increased risk for hospitalization for HF.[28] Whether a reduction in serum SUA can improve cardiac function in T2DM patients with renal dysfunction and/or suboptimal diabetic control merits further evaluation.

\section{Limitation}


Similar to other cross-sectional studies, a causative relation between a high SUA and biventricular myocardial dysfunction was not established in our patients. Nonetheless an SUA lowering agent has previously been proven to reduce hospitalization for $\mathrm{HF}$ [2], suggesting that hyperuricemia contributes to the development of myocardial dysfunction. Our patients were asymptomatic so did not undergo routine anatomical or functional assessment for underlying ischemia and the present international recommendation does not encourage routine screening for underlying coronary artery disease in patients with T2DM.[29] Our study population who continued their normal daily routine nonetheless demonstrated a strong correlation of SUA with myocardial dysfunction so the result may apply to all T2DM patients routinely seen in our clinics.

\section{Conclusion}

Our result demonstrates a close relation between hyperuricemia and biventricular myocardial dysfunction in patients with T2DM. This association persisted in those with and without renal dysfunction and $\mathrm{HbA} 1 \mathrm{c}<$ and $\geq 7 \%$. The potential of a uric acid lowering agent prescribed to patients with T2DM and concomitant hyperuricemia to prevent myocardial dysfunction merits elucidation by future randomized study.

\section{List Of Abbreviations}

A, peak velocity in late diastole of mitral valve inflow

BMI, body mass index

CS, circumferential strain

eGFR, estimated glomerular filtration rate

$E$, peak velocity in early diastole of mitral valve inflow

E'-lat, early diastolic velocity of mitral valve annulus at lateral

E'-sep, early diastolic velocity of mitral valve annulus at septum

FBG, fasting blood glucose

GLS, global longitudinal strain

HbA1c, glycated hemoglobin

HDL-c, high-density lipoprotein cholesterol

IVSd, inter-ventricular septal dimension at end-diastole

LDL-c, low-density lipoprotein cholesterol

LV, Left ventricular

LVEDd, LV end-diastolic dimension

LVEDV, LV volume at the end of diastolic

LVEF, LV ejection fraction

LVESV, LV volume at the end of systolic

Page 12/16 
LVMI, LV mass index

LVPWd, LV posterior wall thickness at end-diastole

MDRD, Modification of Diet in Renal Disease

$\mathrm{RS}$, radial strain

$\mathrm{RV}$, right ventricular

RVEDA, RV end-diastolic area

RVESA, RV end-systolic area

RV-FAC, RV fractional area change

RV-FWLS, right ventricular free wall longitudinal strain

RVSP, RV systolic pressure

RWT, relative wall thickness

SUA, serum uric acid

T2DM, type 2 diabetes mellitus

TAPSE, tricuspid annular plane systolic excursion

$\mathrm{TC}$, total cholesterol

TG, total triglyceride.

\section{Declarations}

\section{Ethics approval and consent to participate}

The study was approved by the ethics committee of the West Cluster Hospital

Authority of Hong Kong, and ethics committee of the University of Hong Kong Shenzhen Hospital, Shenzhen, Chia. All participants gave written informed consent prior to any study-related procedures.

\section{Consent for publication}

Not applicable

\section{Availability of data and materials}

The datasets generated and/or analysed during the current study are available from the corresponding author on reasonable request.

\section{Competing interests}


The authors declare that they have no conflict of interest.

\section{Funding}

This study was supported by HKU-SZH Fund for Shenzhen Key Medical

Discipline (No. SZXK2020081), and Sanming Project of

Cardiology, the university of Hong Kong Shenzhen hospital, Sanming grant from the Ministry of Health, Shenzhen, China (No. SZSM201911020).

\section{Authors' Contributions}

Ju-Hua Liu contributed to the study conception and design, performed data acquisition and analysis of data, write and revise the manuscript. Mei-Zhen Wu, Si-Min Li, Yan Chen, Qing-Wen Ren, Qing-Shan Lin and Ming-Yen Ng performed study conception and design, as well as data acquisition and analysis. Hung-Fat Tse made substantial contributions to the study conception and design, and critically revised the manuscript. Kai-Hang Yiu initialed and supervised the study, had full access to all the data and took responsibility for the integrity of data and accuracy of the data analysis in this study. All authors approved the final version to be published.

\section{Acknowledgments}

We thank the medical and nursing staff of Cardiology Division, Queen Mary

Hospital and University of Hong Kong Shenzhen Hospital, for their help and support during this study.

\section{References}

1. Cho NH, Shaw JE, Karuranga S, Huang Y, da Rocha Fernandes JD, Ohlrogge AW et al. IDF Diabetes Atlas: Global estimates of diabetes prevalence for 2017 and projections for 2045. Diabetes Res Clin Pract 2018, 138:271-281.

2. Thanassoulis G, Brophy JM, Richard H, Pilote L. Gout, allopurinol use, and heart failure outcomes. Arch Intern Med 2010, 170:1358-1364.

3. Lejeune S, Roy C, Ciocea V, Slimani A, de Meester C, Amzulescu M et al. Right Ventricular Global Longitudinal Strain and Outcomes in Heart Failure with Preserved Ejection Fraction. J Am Soc Echocardiogr 2020, 33:973-984.

4. Seo J, Jung IH, Park JH, Kim GS, Lee HY, Byun YS et al. The prognostic value of 2D strain in assessment of the right ventricle in patients with dilated cardiomyopathy. Eur Heart J Cardiovasc Imaging 2019, 20:1043-1050.

5. Nochioka K, Querejeta Roca G, Claggett B, Biering-Sørensen T, Matsushita K, Hung CL et al.Right Ventricular Function, Right Ventricular-Pulmonary Artery Coupling, and Heart Failure Risk in 4 US Communities: The Atherosclerosis Risk in Communities (ARIC) Study. JAMA Cardiol 2018, 3:939-948.

6. 2. Classification and Diagnosis of Diabetes: Standards of Medical Care in Diabetes-2019. Diabetes Care 2019, 42(Suppl 1):S13-s28.

7. Stevens LA, Coresh J, Greene T, Levey AS. Assessing kidney function-measured and estimated glomerular filtration rate. N Engl J Med 2006, 354(23):2473-2483.

8. Lang RM, Badano LP, Mor-Avi V, Afilalo J, Armstrong A, Ernande L et al. Recommendations for cardiac chamber quantification by echocardiography in adults: an update from the American Society of Echocardiography and the European Association of Cardiovascular Imaging. Eur Heart J Cardiovasc Imaging 2015, 16(3):233-270.

Page $14 / 16$ 
9. Nagueh SF, Smiseth OA, Appleton CP, Byrd BF, 3rd, Dokainish H, Edvardsen T et al. Recommendations for the Evaluation of Left Ventricular Diastolic Function by Echocardiography: An Update from the American Society of Echocardiography and the European Association of Cardiovascular Imaging. J Am Soc Echocardiogr 2016, 29(4):277314.

10. Rudski LG, Lai WW, Afilalo J, Hua L, Handschumacher MD, Chandrasekaran K et al. Guidelines for the echocardiographic assessment of the right heart in adults: a report from the American Society of Echocardiography endorsed by the European Association of Echocardiography, a registered branch of the European Society of Cardiology, and the Canadian Society of Echocardiography. J Am Soc Echocardiogr 2010, 23(7):685-713; quiz 786688.

11. Chan J, Shiino K, Obonyo NG, Hanna J, Chamberlain R, Small A et al. Left Ventricular Global Strain Analysis by TwoDimensional Speckle-Tracking Echocardiography: The Learning Curve. J Am Soc Echocardiogr 2017, 30(11):10811090.

12. Stolfo D, Albani S, Biondi F, De Luca A, Barbati G, Howard L et al. Global Right Heart Assessment with SpeckleTracking Imaging Improves the Risk Prediction of a Validated Scoring System in Pulmonary Arterial Hypertension. J Am Soc Echocardiogr 2020, 33(11):1334-1344.e1332.

13. Jia G, DeMarco VG, Sowers JR. Insulin resistance and hyperinsulinaemia in diabetic cardiomyopathy. Nat Rev Endocrinol 2018, 12:144-153.

14. Marwick TH, Ritchie R, Shaw JE, Kaye D. Implications of Underlying Mechanisms for the Recognition and Management of Diabetic Cardiomyopathy. J Am Coll Cardiol 2018, 71:339-351.

15. Zhao CT, Wang M, Siu CW, Hou YL, Wang T, Tse HF et al. Myocardial Dysfunction in Patients With Type 2 Diabetes Mellitus: Role of Endothelial Progenitor Cells and Oxidative Stress. Cardiovasc Diabetol 2012, 11:147.

16. Sacre JW, Franjic B, Jellis CL, Jenkins C, Coombes JS, Marwick TH. Association of Cardiac Autonomic Neuropathy With Subclinical Myocardial Dysfunction in Type 2 Diabetes. JACC Cardiovasc Imaging 2010, 3:1207-1215.

17. Moir S, Hanekom L, Fang ZY, Haluska B, Wong C, Burgess M et al. Relationship Between Myocardial Perfusion and Dysfunction in Diabetic Cardiomyopathy: A Study of Quantitative Contrast Echocardiography and Strain Rate Imaging. heart 2006, 92:1414-1419.

18. De Jong KA, Czeczor JK, Sithara S, McEwen K, Lopaschuk GD, Appelbe A et al. Obesity and type 2 diabetes have additive effects on left ventricular remodelling in normotensive patients-a cross sectional study. Cardiovasc Diabetol $2017,16: 21$.

19. Zoppini G, Bergamini C, Bonapace S, Rossi A, Trombetta M, Mantovani A et al. Association Between Subclinical Left Ventricular Systolic Dysfunction and Glycemic Control in Asymptomatic Type 2 Diabetic Patients With Preserved Left Ventricular Function. J Diabetes Complications 2017, 31:1035-1040.

20. Nakanishi K, Daimon M, Yoshida Y, Ishiwata J, Sawada N, Hirokawa M et al. Serum uric acid level and subclinical left ventricular dysfunction: a community-based cohort study. ESC Heart Fail 2020, 7:1031-1038.

21. Fang $X$, Pan $C$, Chen $Y$, Sun $M$, Zhang Z, Jiang $L$ et al. Assessment of subclinical left ventricular changes in essential hypertensive patients with hyperuricemia: A three-dimensional speckle-tracking echocardiography study. Clin Exp Hypertens 2017, 39:93-99.

22. Bergamini C, Cicoira M, Rossi A, Vassanelli C. Oxidative stress and hyperuricaemia: pathophysiology, clinical relevance, and therapeutic implications in chronic heart failure. Eur J Heart Fail 2009, 11:444-452.

23. Leyva F, Anker SD, Godsland IF, Teixeira M, Hellewell PG, Kox WJ et al. Uric acid in chronic heart failure: a marker of chronic inflammation. Eur Heart J 1998, 19:1814-1822.

24. Gullu H, Erdogan D, Caliskan M, Tok D, Kulaksizoglu S, Yildirir A et al. Elevated serum uric acid levels impair coronary microvascular function in patients with idiopathic dilated cardiomyopathy. Eur J Heart Fail 2007, 9:466-468. 
25. Longobardo L, Suma V, Jain R, Carerj S, Zito C, Zwicke DL et al. Role of Two-Dimensional Speckle-Tracking Echocardiography Strain in the Assessment of Right Ventricular Systolic Function and Comparison with Conventional Parameters. J Am Soc Echocardiogr 2017, 30:937-946.

26. Tadic M, Celic V, Cuspidi C, llic S, Pencic B, Radojkovic J et al. Right heart mechanics in untreated normotensive patients with prediabetes and type 2 diabetes mellitus: a two- and three-dimensional echocardiographic study. J Am Soc Echocardiogr 2015, 28:317-327.

27. Zhao W, Katzmarzyk PT, Horswell R, Wang Y, Johnson J, Hu G. HbA1c and heart failure risk among diabetic patients. J Clin Endocrinol Metab 2014, 99:E263-267.

28. Lind M, Olsson M, Rosengren A, Svensson AM, Bounias I, Gudbjörnsdottir S. The relationship between glycaemic control and heart failure in 83,021 patients with type 2 diabetes. Diabetologia 2012, 55:2946-2953.

29. Arnett DK, Blumenthal RS, Albert MA, Buroker AB, Goldberger ZD, Hahn EJ et al. 2019 ACC/AHA Guideline on the Primary Prevention of Cardiovascular Disease: Executive Summary: A Report of the American College of Cardiology/American Heart Association Task Force on Clinical Practice Guidelines. J Am Coll Cardiol 2019, 74(10):1376-1414.

\section{Supplementary Files}

This is a list of supplementary files associated with this preprint. Click to download.

- SupplementaryTables.docx 